\title{
Partially-erupting prominences: a comparison between observations and model-predicted observables
}

\author{
D. Tripathi ${ }^{1}$, S. E. Gibson ${ }^{2}$, J. Qiu ${ }^{3}$, L. Fletcher ${ }^{4}$, R.Liu ${ }^{5}$, H. Gilbert ${ }^{5}$, and H. E. Mason ${ }^{1}$ \\ 1 Department of Applied Mathematics and Theoretical Physics, Wilberforce Road, Cambridge CB3 0WA, UK \\ e-mail: [D.Tripathi;H.E.Mason] adamtp.cam.ac.uk \\ 2 High Altitude Observatory, National Center of Atmospheric Research, Boulder, Colorado, USA \\ e-mail: sgibson@ucar.edu \\ 3 Montana State University, Bozeman, MT 59717-3840, USA \\ e-mail: qiuj@mithra.physics.montana.edu \\ 4 University of Glasgow, Glagow G12 8QQ, UK \\ e-mail: lyndsay@astro.gla.ac.uk \\ 5 Department of Physics and Astronomy, Rice University, 6100 Main St., Houston, TX 77005, USA \\ e-mail: [hgilbert;rliu] @rice.edu
}

Received 18 March 2008 / Accepted 6 February 2009

\begin{abstract}
Aims. We investigate several partially-erupting prominences to study their relationship with other CME-associated phenomena and compare these observations with observables predicted by a model of partially-expelled-flux-ropes (Gibson \& Fan 2006a, ApJ, 637, L65; 2006b, J. Geophys. Res., 111, 12103).

Methods. We studied 6 selected events with partially-erupting prominences using multi-wavelength observations recorded by the Extreme-ultraviolet Imaging Telescope (EIT), Transition Region and Coronal Explorer (TRACE), Mauna Loa Solar Observatory (MLSO), Big Bear Solar Observatory (BBSO), and Soft X-ray Telescope (SXT). The observational features associated with partiallyerupting prominences were then compared with the predicted observables from the model.

Results. The partially-expelled-flux-rope (PEFR) model can explain the partial eruption of these prominences, and in addition predicts a variety of other CME-related observables that provide evidence of internal reconnection during eruption. We find that all of the partially-erupting prominences studied in this paper exhibit indirect evidence of internal reconnection. Moreover, all cases showed evidence of at least one observable unique to the PEFR model, e.g., dimmings external to the source region and/or a soft X-ray cusp overlying a reformed sigmoid.

Conclusions. The PEFR model provides a plausible mechanism to explain the observed evolution of partially-erupting-prominenceassociated CMEs in our study.
\end{abstract}

Key words. Sun: corona - Sun: coronal mass ejections (CMEs) - Sun: prominences - Sun: filaments

\section{Introduction}

Coronal mass ejections (CMEs) are routinely interpreted as possessing a helical magnetic flux rope structure (see e.g. Chen et al. 1997; Dere et al. 1999; Plunkett et al. 2000). Magnetic clouds, i.e., interplanetary structures that have been shown to be associated with CMEs, are also interpreted to be magnetic flux ropes (Burlaga et al. 1981, 1982; Burlaga 1988). An ongoing controversy remains, however, as to whether a precursor flux rope exists as a coronal equilibrium state prior to eruption or whether it is formed during eruption. This is an important question to resolve, since CME initiation models and space weather predictions depend upon a clear understanding of the configuration of pre-CME magnetic fields and their evolution during eruption.

The existence of a precursor magnetic flux rope is an attractive concept from a theoretical point of view, as it may represent a minimum magnetic energy configuration (Taylor 1974; Low 1996, 1999; Rust 2003; Janse \& Low 2007). A precursor flux rope has also been used to explain a wide range of pre-CME phenomena, including photospheric magnetic flux evolution (Lites et al. 1995; López Fuentes et al. 2000;
Green et al. 2001; Fan 2001; Mandrini et al. 2002; Gibson et al. 2004) and prominences, associated white-light cavities, and soft X-ray sigmoids (Priest et al. 1989; Rust \& Kumar 1994; Aulanier \& Demoulin 1998; Amari et al. 1999; Gibson et al. 2004; van Ballegooijen 2004; Gibson \& Fan 2006b). For CMEs, where white-light, low coronal observations are available and which are unobscured by unrelated features along the line of sight, the prominence and its cavity have been tracked from preeruption through their expansion outwards in the CME (Fisher \& Poland 1981; Illing \& Hundhausen 1985; Hundhausen 1999; Srivastava et al. 1999; Maričić et al. 2004; Gibson et al. 2006b). In many cases, a flux rope model can be used to match the magnetic flux and chirality of precursor structures to magnetic clouds (Bothmer \& Rust 1997; Bothmer \& Schwenn 1997; Rust et al. 2005).

On the other hand, observations associated with flares demonstrate the importance of magnetic reconnection in the eruption, and challenge the picture of a pre-existing flux rope simply losing equilibrium and expanding out into interplanetary space. Such a purely ideal eruption is inconsistent with observations which find that the impulsive stage of the flare is linked 
to that of maximum $\mathrm{CME}$ acceleration, implying that reconnections are significant to the dynamic evolution of the CME (Zhang et al. 2001; Tripathi et al. 2006a; Chifor et al. 2006; Zhang \& Dere 2006; Chifor et al. 2007). Most models of an erupting (pre-existing) flux rope do involve significant magnetic reconnections at a current sheet below the rope which serve to "close down" the field beneath it (e.g., Lin et al. 1998), and are consistent with post-eruption arcade formations and flareCME timing observations (see e.g., Tripathi et al. 2004; Tripathi 2006). However, the field lines of the rope itself may not be involved in these reconnections (see Amari et al. 2003, for an exception). Observations of soft X-ray loops as well as of chromospheric flare ribbons indicate that reconnection occurs initially along highly sheared loops and only later do the magnetic field lines make a transition to more potential arcade loops (Canfield et al. 2000; Martin \& McAllister 1995; Su et al. 2006) in a manner explained by models where reconnections take place initially in a sheared magnetic core (e.g., Moore et al. 1997). A further analysis of flare ribbons implies that the bulk of magnetic cloud poloidal flux originates in reconnecting field lines (Qiu et al. 2007), and studies of magnetic cloud charge states indicate possibly flare-associated heating along prominence-mass-carrying field lines (Skoug et al. 1999; Gloeckler et al. 1999; Reinard 2005). These observations indicate that the flux rope that escapes in the $\mathrm{CME}$ is made up of field lines that have undergone significant reconnection, as would be the case if the flux ropes were formed in situ during eruption, but which would not be the case for a pre-existing rope expanding in its entirety upwards without significant change of topology/connectivity.

Neither a totally erupting, pre-existing rope, nor a rope that forms completely in situ during eruption can explain the full range of these observations. An alternative to either of these extremes is a "partially-expelled flux rope" (PEFR) model (Gibson \& Fan 2006a). In this model, a flux rope exists prior to the CME, plays an essential role in triggering it (Fan 2005), and also explains a range of pre-CME observations of prominences, cavities, and sigmoids (Gibson \& Fan 2006b; Gibson et al. 2006a), as well as post-eruption phenomena such as coronal dimmings and the structure of magnetic clouds (Gibson \& Fan 2008). However, as it erupts, it reconnects internally and with surrounding fields so that it breaks in two, with one portion of the rope remaining behind, and the other escaping as the CME and magnetic cloud.

It is very common for a significant portion of prominence mass to remain behind after an eruption (see e.g., Gilbert et al. 2000). Because prominence mass is often modeled as being situated within the dips of a magnetic flux rope, one possibility is that such "partially-erupting prominences" occur because of a flux-rope bifurcation as predicted by the model. It is often difficult to tell, however, whether internal reconnections are indeed occurring, or whether the apparent split in the prominence mass arises from the differing evolution of adjacent, but magneticallydisconnected structures. It is therefore essential to consider a range of multi-wavelength observations in order to look for additional evidence of internal reconnection consistent with predicted model observables. It is worth emphasizing here that it is almost impossible to observe reconnection directly in the corona as there is no direct measurement of magnetic field in the corona. Therefore, we have to rely on indirect evidence derived from the multi-wavelength observations.

The rest of the paper is structured as follows. In Sect. 2 we will describe the observables predicted by the model. In Sect. 3 we will present analyses of cases of partially erupting prominences, and consider how well the data supports the model. In Sect. 4 we present our conclusions.

\section{Bifurcating magnetic flux ropes: PEFR model-predicted observables}

The concept of a flux rope breaking in two during eruption was first discussed by Gilbert et al. (2000), as a means of explaining observations where only a portion of the pre-eruption prominence escaped in the eruption (note that a filament and prominence are the same entity, differing only in how they are observed: we will use the terms interchangeably from here on). These authors find that the majority of erupting prominences studied demonstrate a separation of escaping material from the bulk of the prominence, and propose that reconnections occurr within the prominence at an X-type neutral line forming during the eruption. In a numerical simulation of the dynamic emergence of a magnetic flux rope across the photosphere, Manchester et al. (2004) demonstrate that shearing motions induced by axial field gradients could lead to internal reconnections, and ultimately the rope's bifurcation and the upward expansion of its upper portion. Birn et al. (2006) likewise demonstrate the formation of a current sheet within an unstable flux rope, which separated an outwardly expanding portion of the rope from a portion that remained below. Gibson \& Fan (2006a) describe the full evolution of such a partially-expelled flux rope (PEFR), from the rope's emergence and formation as a pre-eruption equilibrium, through its destabilization, eruption, and bifurcation, and ultimately to an end-state with magnetic field closing down over the surviving portion of the rope. This is the PEFR model we will specifically consider in this paper. We now summarize the model predictions for observables that can be directly compared to data (see Gibson \& Fan 2006b, 2008; and Gibson et al. 2006a, for further details).

The first set of observables provide evidence of a partial eruption of prominence mass:

- Ejected prominence mass: Fig. 1 shows the evolution of initially dipped field (brown) during the rope's eruption, which we identify with the prominence mass. Current sheets form within the rope, splitting the erupting material in two (visible in the right-hand image as the central thin, vertical density enhancement above the reformed cavity). The uppermost material escapes upwards, and is the core of the the three-part (dome/cavity/core) structure of the CME. See also Figs. 5 and 8 of Gibson \& Fan (2006b).

- Surviving prominence mass: in Fig. 1 right-hand image, the brown material lying below the central current sheet is essentially unaffected by the eruption. Thus, some portion of prominence mass is not ejected.

Because adjacent, magnetically disconnected structures might create the illusion of partial eruption, it is important to consider the next two sets of observables, which provide evidence of internal reconnection:

- X-type flows: the prominence-tracing material splits in two because of reconnection at the central current sheet (see Fig. 5 in Gibson \& Fan 2006b). The model therefore predicts mass flows diverging from a central point, so that the upper material would continuously move out, while material below the reconnection point might first surge up, but then fall back down.

- Two ribbon flares surrounding non-erupting filament: Fig. 2 (left panel) shows the footpoints of field lines that have reconnected at the current sheets, with the surviving filament 

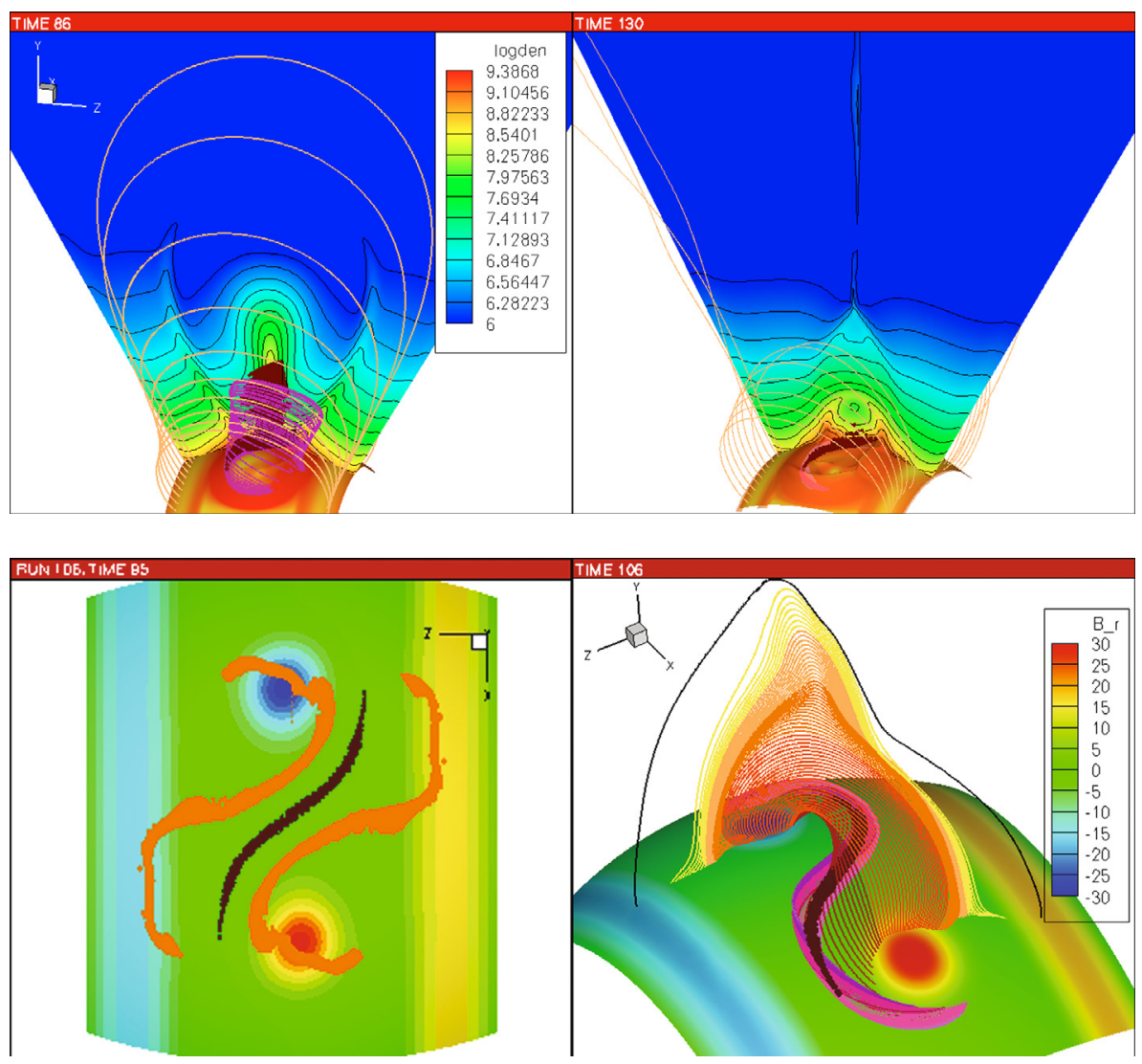

Fig. 1. PEFR-model partially-erupting prominence and reforming cavity. Isosurface and isocontours show the logarithm of number density (in cgs units), magenta lines show the baldpatch-separatrix-surface (BPSS) (see text and Fig. 2 of Gibson \& Fan 2006b), and yellow lines show overlying arcade field. Evolving, initially-dipped field identified with the prominence is shown in brown.

Fig. 2. Left: footpoints of reconnected field lines (orange) forming two ribbons surrounding surviving portion of filament (brown). See Gibson \& Fan (2006a) for discussion of how reconnected field lines are determined. Right: adopted from Gibson \& Fan (2006b) Posteruption state: cusp over sigmoid and filament. Red-orange-black field lines are sample lines straddling the magnetic neutral line lines show bald-patch-separatrix-surface (BPSS) of surviving rope, brown shows surviving portion of filament. shown in brown. Thus, a two-ribbon flare would bracket the surviving portion of the filament, and indicate reconnection above it. Since this signature is also consistent with completely non-erupting filament e.g., in case of confined flares, it is important to combine it with evidence of ejected filament mass as described above.

Internal reconnection within sheared (but not flux-rope) precursor fields could explain all of the observables listed so far (see e.g., Tokman \& Bellan 2002). The observables we now list, however, are unique to the PEFR model:

- Transition from sigmoid to cusp-overlying-sigmoid state: the transition from sheared to cusp-shaped arises as reconnections occur initially at the sigmoidal surface separating rope and arcade, then on sigmoidal lines within the core of the rope, and finally behind the erupting portion of the rope forming the cusp (see bottom panel of Fig. 5 in Gibson \& Fan 2006b). Such a transition would also occur for non-fluxrope-precursor models, but the PEFR model makes the additional prediction that a sigmoid would reform below the cusp after the eruption. This is shown in the right panel of Fig. 2, where the surviving portion of the flux rope is illustrated by the magenta field lines which represent the critical "bald-patch-separatrix-surface" (BPSS) of dipped field just grazing the "photosphere" (i.e., the simulation's lower boundary). The BPSS arises from the flux rope topology, and has been demonstrated to be a site where current sheets form under perturbation - not just during eruption - and so may explain "quiescent" (non-eruptive) sigmoids. The predicted observable of the PEFR model is thus a sigmoid transitioning to a cusp which overlays a quickly reforming quiescent sigmoid.

- Transient coronal holes rooted outside original source region: another type of reconnection explicitly predicted by the PEFR model is connectivity-changing reconnections between the rope and the arcade (see bottom panel of Fig. 5 in Gibson \& Fan 2006b). These "mixed-connectivity" field lines possess higher, erupting counterparts, which undergo further "rope-breaking" reconnections at the central, vertical sheets to result in an escaping rope which is rooted in the original arcade boundary. If transient coronal holes correspond to the footpoints of the escaping flux rope, the PEFR model predicts that they would lie completely outside the original source region (see Fig. 8 in Gibson \& Fan 2008).

- Pre-existing and reforming cavity (subject to line-of-sight visibility): note that the PEFR model also predicts that both the initial, and the surviving filaments are contained within a region of decreased density, i.e. a cavity (Fig. 1). The cavity is an integral part of a flux rope model, with a sharplydefined circular boundary arising from a magnetic flux surface (Low 1996, 1999). Thus, the survival of the lower flux rope would predict a reformation of the cavity after the eruption. Note, however, that such a reforming cavity would only be likely to be observed for partial eruptions of nearlimb, large-scale-prominences without significant intervening structures. Although partially-erupting filament/cavity systems have been reported (e.g. Liu et al. 2007), in such cases it is particularly difficult to rule out the eruption of adjacent structures along the line of sight. Because the observables providing evidence of internal reconnection tend to be best viewed on-disk, the events studied in this paper do not allow us to look for evidence of a reforming cavity.

\section{Observations and analysis}

The model predicts partially-erupting filaments, so the first test of its plausibility is to consider how common they may be. We find, as Gilbert et al. (2000) did, that some sort of splitting of 

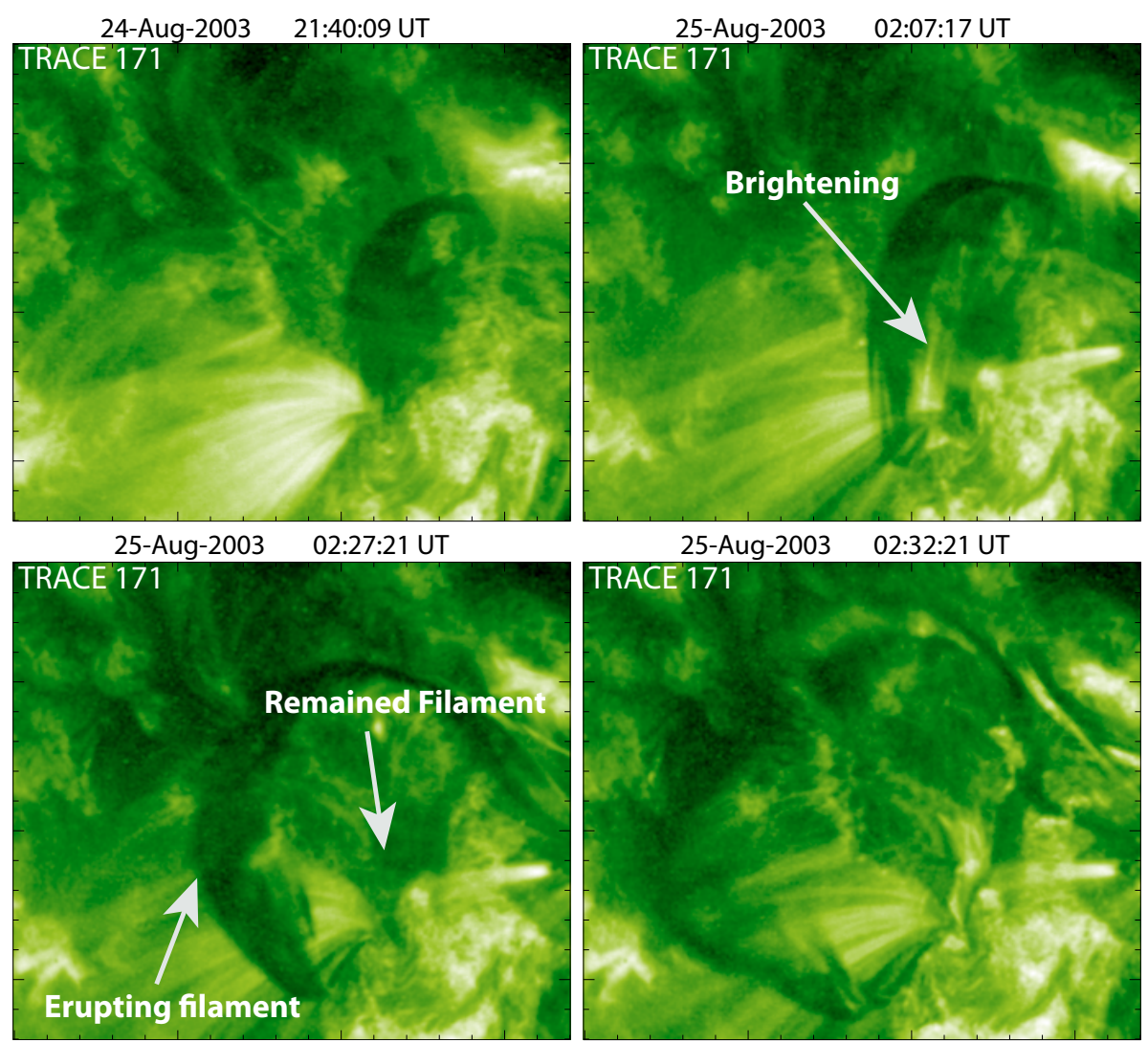

Fig. 3. TRACE observation of a filament breaking while eruption on 25-Aug.-2003. The brightening, erupting and remaining filament are marked with arrows and labeled.

prominence material occurs in many, if not most, cases of eruption. In some cases, a prominence is seen at a viewing angle from which it appears to rise as a whole, and subsequently breaks in two with respect to its height, e.g. Fig. 3. Such cases, particularly when coupled with brightenings and/or diverging flows at the break points, are convincing examples of filaments breaking at an internal reconnection point (see, e.g., Tripathi et al. 2006b, 2007). However, other eruptions happen along the length of a filament, e.g., Fig. 4. Although these too could be due to internal reconnection, one can not generally rule out the possibility that the eruption separated two structures that were not magneticallyconnected to begin with.

It is also important to pay close attention to time scales of the filament's dynamic evolution. Two-thirds of erupting filaments reform in the same place and with much the same shape within 1 to 7 days (Priest 1984). Moreover, filaments are likely to be heated during eruption so that they may temporarily leave the $\mathrm{H} \alpha$ bandpass. If so, they might be better seen in EUV or SXR observations. Hence, multi-wavelength observations are important in establishing the timing and extent of filament eruptions.

In choosing our partial-eruption cases, therefore, we require the following:

1. ejection of filament material, in particular the presence of a core within the associated CME;

2. survival of material, in particular the reappearance of a filament in $\mathrm{H} \alpha$ and/or EUV;

3. evidence of internal reconnection, as described in the model observables above.

\subsection{Event on April 29, 1998}

An erupting filament associated with an M-class flare was observed on 29-Apr.-1998. The eruption was also observed by the
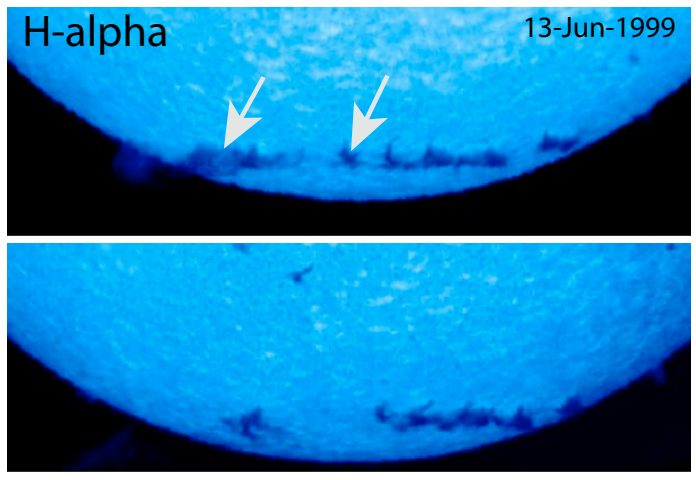

Fig. 4. BBSO $\mathrm{H} \alpha$ Observation of an erupting polar crown filament on 13-Jun.-1999. The part of the filament which erupts is marked by an arrow.

BBSO H $\alpha$ telescope (top row images in Fig. 5), the EIT at $195 \AA$ (middle row in Fig. 5), and by the SXT (bottom row in Fig. 5).

Evidence of both ejected and surviving material: the filament eruption was associated with a halo CME as observed by the LASCO/C2 coronagraph at 16:58:54 UT. Although the CME was a halo, a white-light core is visible in the structure which can be associated with the erupting filament, that was also visible as ejected material in EIT running difference observations. The top panel in Fig. 5 displays images of the erupting filament and associated flare as observed in $\mathrm{H} \alpha$. A dark filamentary structure, marked with an arrow in the last image, is located at the same position as that of the original filament before the eruption, providing evidence of surviving material.

Evidence of internal reconnection (non-PEFR unique): the chromospheric counterparts of the flare are two ribbons, seen in the top panel images with the dark filament in between. The middle image is recorded at around the peak of the flare. As the 


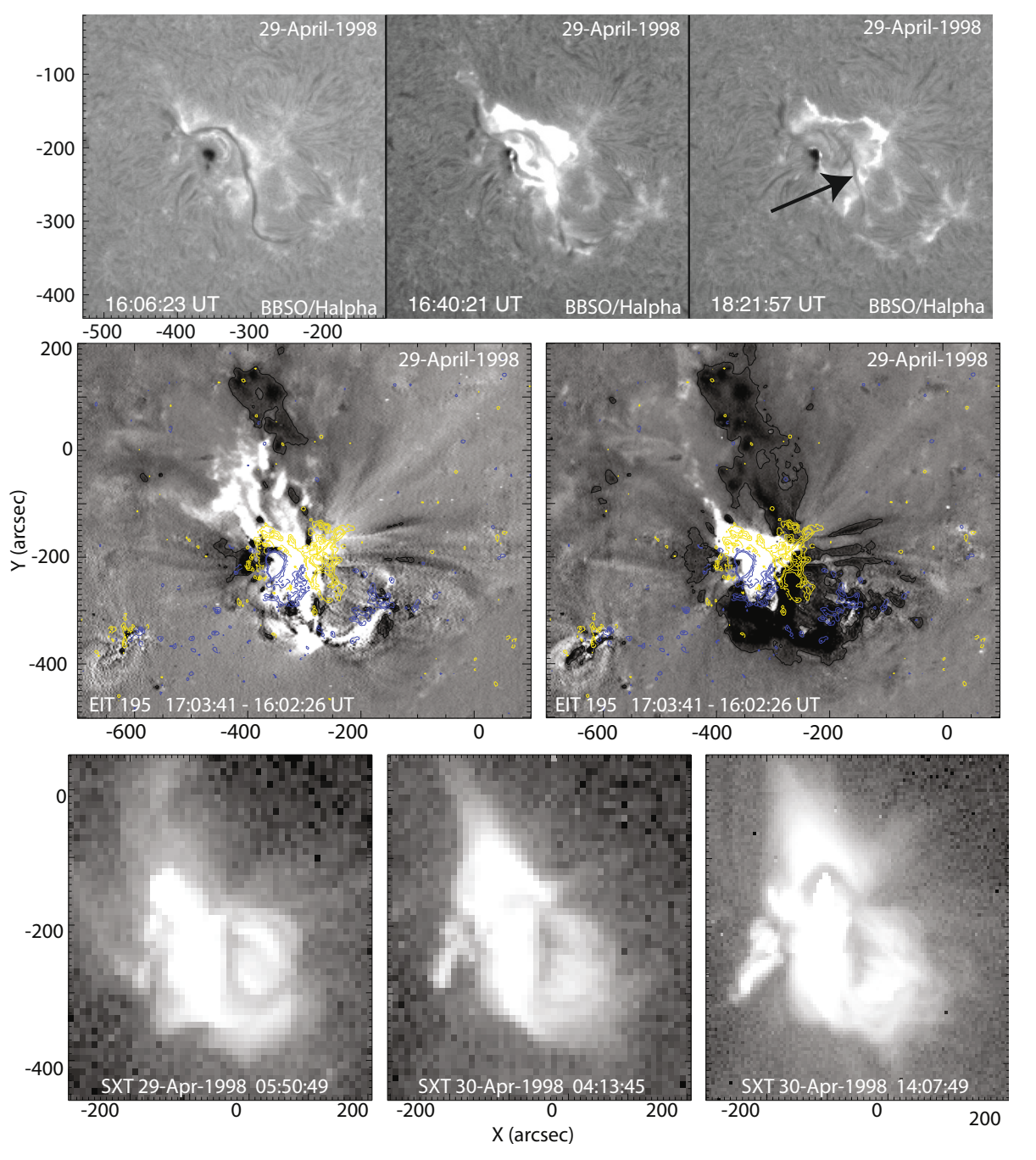

Fig. 5. Top panel: $\mathrm{H} \alpha$ images taken from BBSO, showing the erupting filament on 29Apr.-1998. The arrow in the last image locates the surviving filament in between two ribbons. Middle panel: base difference images taken by EIT at $195 \AA$ on 29-Apr.-1998. Note that the image recorded by EIT at 16:02 UT was considered as the base image and the images were differentially rotated to line up with the base image prior to the subtraction. Overplotted black contours show the dimming regions (indicating a $40 \%$ decrease in the intensity relative to the base image). Yellow contours are positive polarity and blue contours represent the negative polarity regions as was observed by the MDI magnetograms. Bottom panel: Yohkoh/SXT images showing the evolution of the source region before and after the eruption, on 29-Apr-1998 (left image) and on 30-Apr.-1998 (middle and right images). ribbons separate outwards from the neutral line, the contrast of the filamentary structure against the flare emission improves and it is seen more clearly (see the last image).

Evidence of observables unique to PEFR model: the bottom panel shows SXR loops: the left image shows a clear sigmoidal system of hot loops overlying the sigmoidal filament seen in the $\mathrm{H} \alpha$ observations. After the eruption the sigmoidal region has transitioned to cusp-shaped post-eruption arcades (PEAs) overlying a sheared system of loops (see the middle and last images in the bottom panel of Fig. 5). The images in the middle row of Fig. 5 displays EIT $195 \AA$ base difference images (in background) in which a fixed image frame was subtracted from the following images. Base difference images provide information about overall changes in the source region with respect to a fixed image frame. In this case, they demonstrate that the dimming regions lie outside the eruption source region.

\subsection{Event on August 17, 1999}

An erupting filament, associated with a C-class flare was observed on 17-Aug.-1999 (Flare start: 14:37 UT; peak: 15:33 UT).

Evidence of both ejected and surviving material: the filament eruption was associated with a CME with a bright core as observed by LASCO/C2 at 15:30:05 UT. The $\mathrm{H} \alpha$ data recorded by BBSO showed a surviving filament about $2.5 \mathrm{~h}$ post-flare (see Fig. 10, 2nd row, 2nd column image in Gibson et al. 2002).
Evidence of internal reconnection (non-PEFR unique): the surviving filament is clearly straddled by two flare ribbons (also seen towards the north, labeled and marked by arrows in Fig. 10, 2nd row, 2nd column image in Gibson et al. 2002). The filament actually became more pronounced during the eruption, and continued to develop and grow with time (see Fig. 10, 3rd row, 2nd column in Gibson et al. 2002).

Evidence of observables unique to PEFR model: the SXT observations recorded a day before eruption showed a clear sigmoidal structure. After the eruption, a cusp-shaped structure lying over a part of the surviving sigmoid was seen (see SXT images in Fig. 10 in Gibson et al. 2002). Figure 6 displays base difference images taken by the EIT, demonstrating that the dimming regions predominantly occur outside the main eruption location (see the right panel).

For further details on this event, see Gibson et al. (2002).

\subsection{Event on September 12, 2000}

A spectacular quiescent filament eruption was observed on 12Sep.-2000 associated with an M-class flare (start: 11:06 UT; peak:11:55 UT).

Evidence of both ejected and surviving material: the eruption was also associated with a three-part structured $\mathrm{CME}$ as observed by LASCO/C2 at 11:54 UT. The top panel in Fig. 7 displays the erupting filament detected by the $\mathrm{H} \alpha$ telescope at Kanzelhoehe Solar Observatory. In the middle image, taken at 

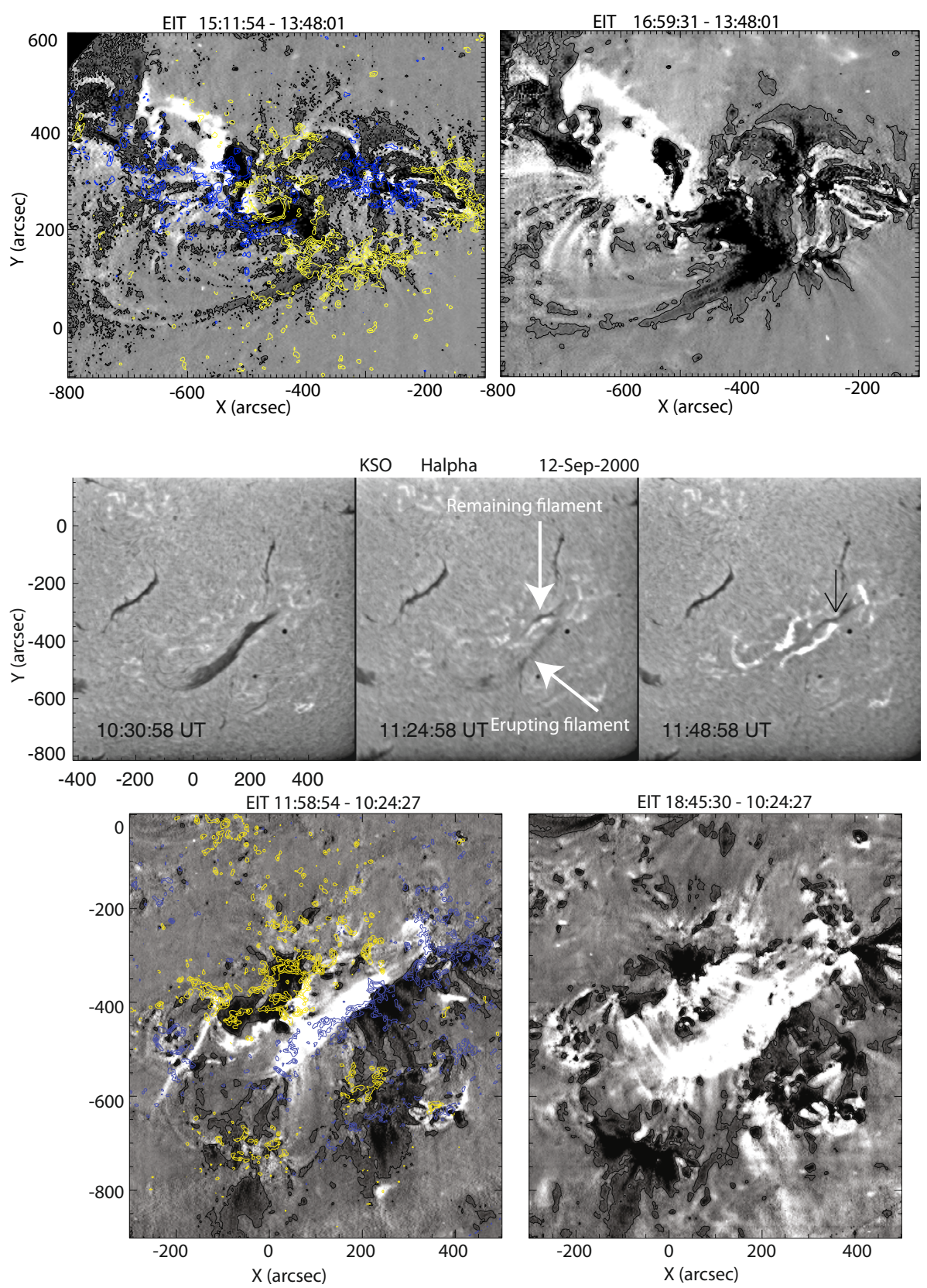

12-Sep-2000
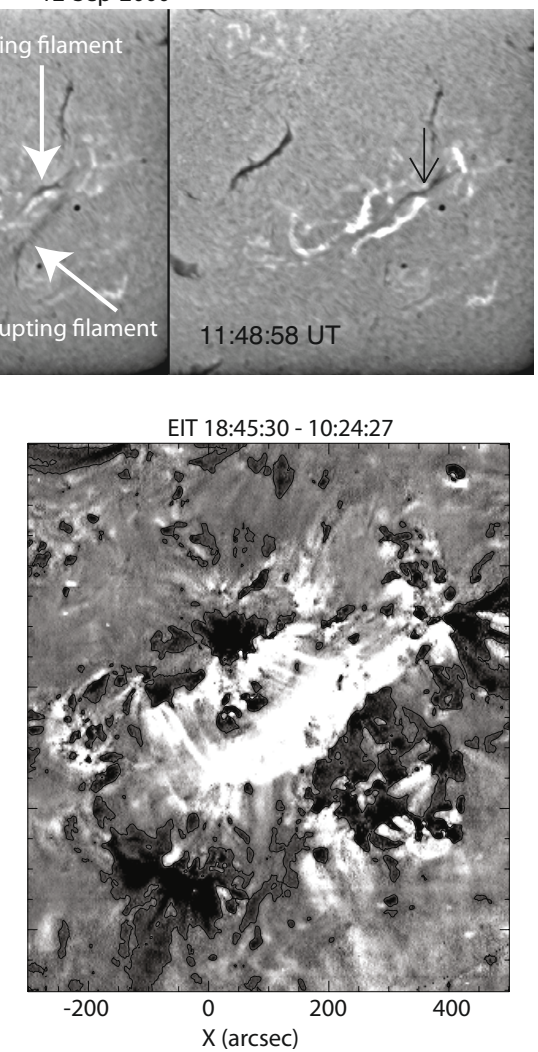

Fig. 6. Base difference images taken by EIT at $195 \AA$ on 17-Aug.-1999 showing dimming regions overplotted with MDI magnetic contours, displayed as in Fig. 5. For this event the base image was taken at 13:48 UT (before the flare).
Fig. 7. Top panels: BBSO $\mathrm{H} \alpha$ images showing the partially erupting filament on 12-Sep.-00. The erupting and surviving filament is marked by arrows and labeled accordingly. Bottom panel: EIT $195 \AA ̊$ A base difference images overplotted with MDI magnetic field contours, displayed as in Fig. 5. For this event the base image was taken at 10:24 UT (before the flare).
11:24 approximately 20 min after the eruption, when the erupting filament is already at some height, a dark filament towards the northwestern side in the source region can be seen. The erupting and the surviving filaments are marked and labeled in the top middle image. This provides evidence that the filament breaks in the middle towards its north-western end during eruption.

Evidence of internal reconnection (non-PEFR unique): the surviving filament is clearly straddled by two flare ribbons (last image in top panel of Fig. 7).

Evidence of observables unique to PEFR model: the bottom panel of Fig. 7 displays the base difference images taken by the EIT at $195 \AA$ overplotted with magnetic field contours. As can be depicted from the figure, the dimming is seen not at the foot points of the erupting filament but in the surrounding region. SXR observations were not available for this event (or others in this paper that post-dated Yohkoh observations and predated Hinode), but the second image in the bottom panel shows the formation of post-eruption arcades which appear to be more sheared towards the north above the surviving filament.

\subsection{Event on June 11, 2003}

An eruption of a filament was observed on 11-Jun.-2003 near the eastern limb.

Evidence of both ejected and surviving material: the top panel in Fig. 8 shows three BBSO/H-alpha images before (left image), during (middle image) and after the eruption (last image). The eastern and western legs of the polar crown filament reform quickly, but its middle part never reforms. A CME with a three-part structure was observed by the MLSO Mk4 coronameter.

Evidence of internal reconnection (non-PEFR unique): filament material is observed to flow back to the Sun's surface during the eruption in both $\mathrm{H} \alpha$ and EIT movies. The bottom three panels in Fig. 8 show BBSO $\mathrm{H} \alpha$ images overlaid with contours 


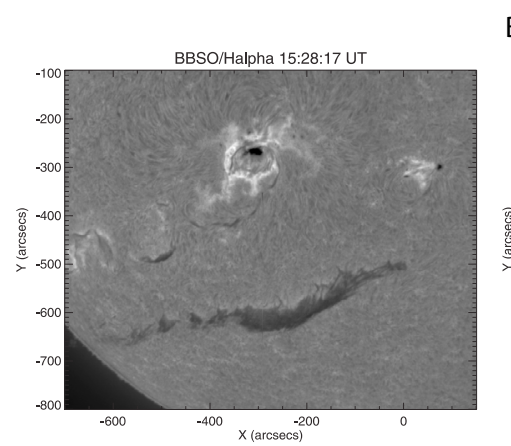

$$
\text { BBSO Halpha 11-Jun-2003 }
$$
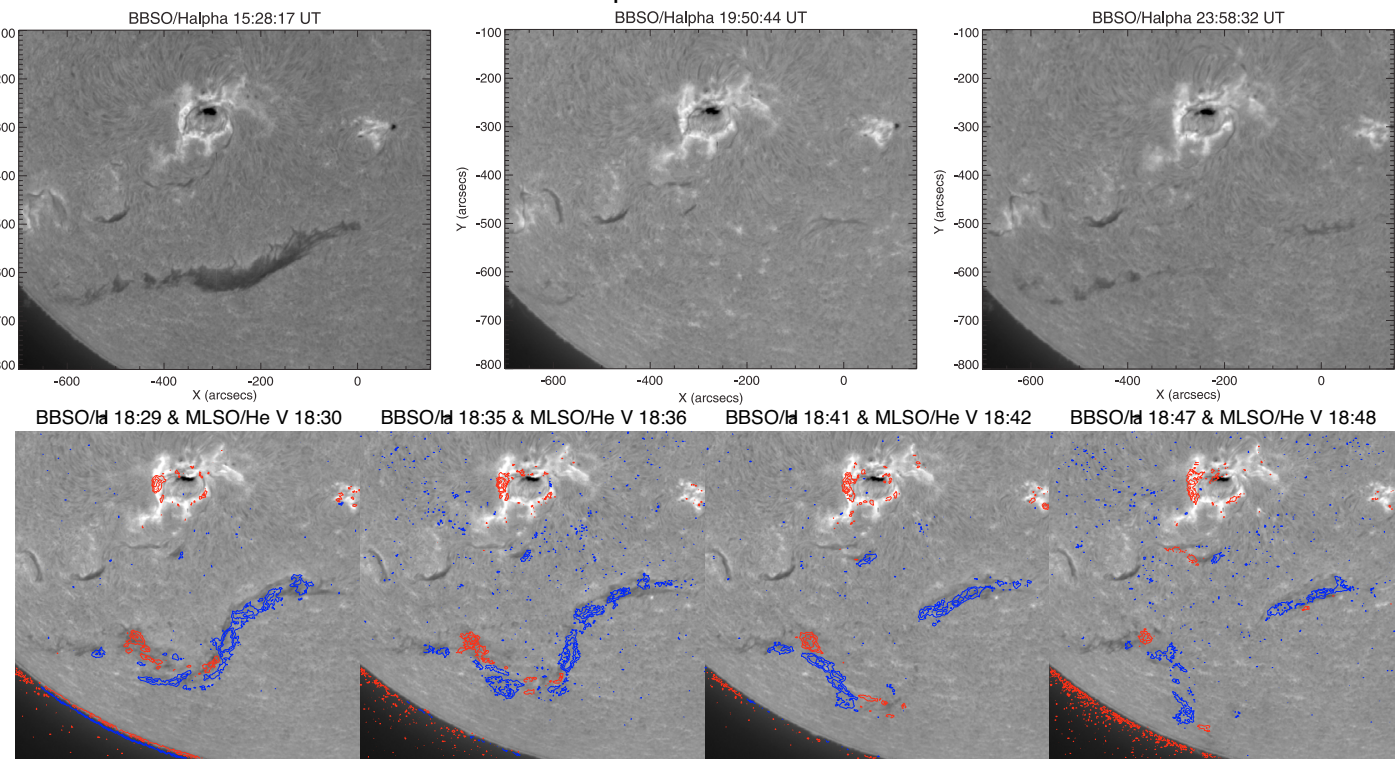

Fig. 8. Top panels: $\mathrm{H} \alpha$ images recorded at BBSO showing the erupting filament (left panel) and filament reformation (right panel) on 11-Jun.-2003. 2nd, 3rd and 4th rows: BBSO H-alpha images overlaid by contours of MLSO/CHIP velocity data on 11-Jun.-2003. Red (blue) contours indicate motions away from (toward) the observer.
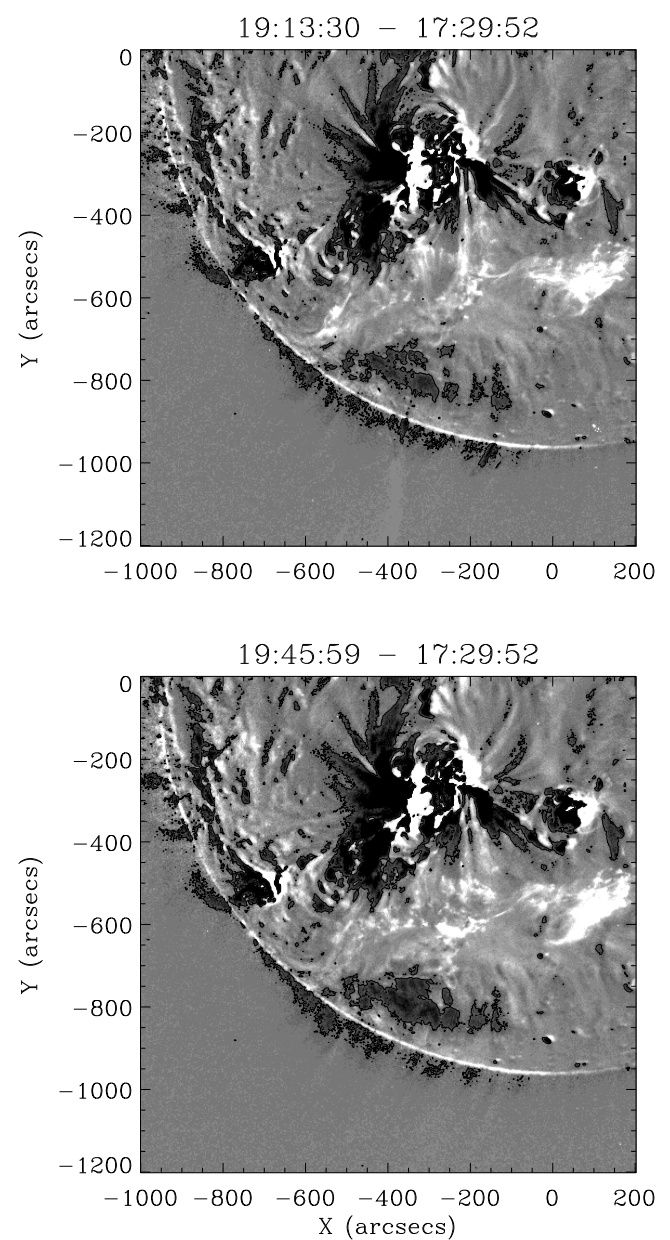

Fig. 9. Base difference images taken by EIT at $195 \AA$ on 11-Jun.-2003.

of MLSO/CHIP velocity data. Red (blue) contours indicate motions away from (toward) the observer. During eruption, the lineof-sight velocity reveals that plasma is moving both towards and away from the Sun being predominantly away, showing that the prominence is moving upward. In the early rise phase of the prominence plasma motion in both directions is evident at the middle of the prominence. Later on, the plasma motion towards the Sun is more dominant towards the eastern leg of the prominence, probably indicating the draining of plasma along the legs.

Evidence of observables unique to PEFR model: Fig. 9 displays base difference images taken by the EIT at $195 \AA$. An image frame taken at 17:29:52 before the eruption was considered as the base image. Since this event was also on the limb, we did not have MDI magnetic field measurements with good sensitivity. However, from Fig. 9, the dimming regions appear to be outside the erupting source regions.

\subsection{Event on August 25, 2003}

A relatively small erupting filament was observed on August 25, 2003 at around 02:00 UT.

Evidence of both ejected and surviving material: the eruption was associated with a white-light $\mathrm{CME}$ comprising a bright core as detected by the LASCO/C2 at 03:25 UT. Figure 3 displays images taken by the TRACE at $171 \AA$. The filament starts with a slow rise phase at around 23:00 UT. At 02:07 UT (top right image in Fig. 3), when the filament has risen some height, it appears to separate in two, leaving some filament material behind. The erupting and surviving filaments are marked with arrows and labeled in the bottom left panel. The top panel (left and middle images) of Fig. 10 display the BBSO $\mathrm{H} \alpha$ filament a day before eruption (marked with arrows). Although surviving material is evident in the TRACE images even during eruption, it was not seen in $H \alpha$ until the next day (top right image of Fig. 10, marked with an arrow). This may be due to heating during eruption, and emphasizes the importance of multi-wavelength observations when identifying the partial eruptions.

Evidence of internal reconnection (non-PEFR unique): a brightening is seen in between the erupting part and the surviving part marked in the top right panel of Fig. 3. this brightening 


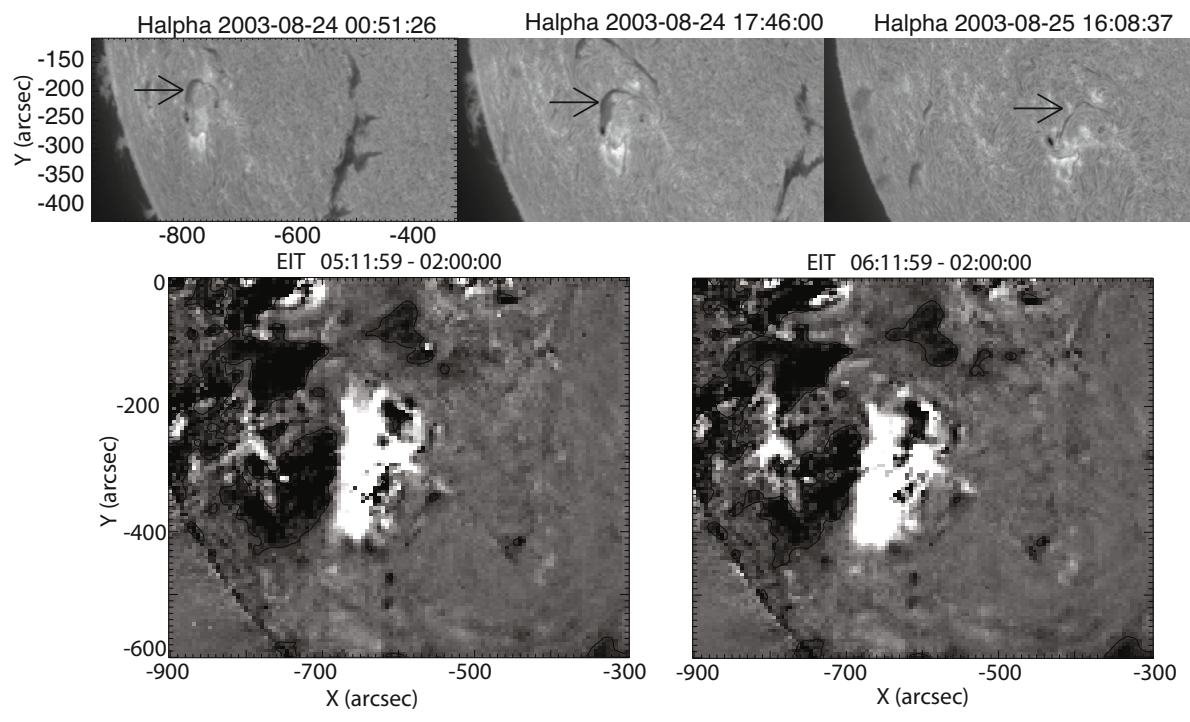

Fig. 10. Top panel: $\mathrm{H} \alpha$ images taken from BBSO, showing the filament before (left and the middle panel) and after the eruption (last panel). Arrows demark the filament which is of interest. Bottom panel: EIT at $195 \AA$ base difference images taken on 25-Aug.-2003 displayed as in Fig. 5. For this event the base image was taken at 02:00 UT (before the flare). may indicate energy release due to reconnections within the erupting filament.

Evidence of observables unique to PEFR model: the bottom panel of Fig. 10 displays EIT $195 \AA$ base difference images. Since this event occurs far from the disk center, we did not have enough sensitivity for magnetic field data. However, the EIT dimming observations on their own demonstrate that the dimmings are predominantly outside the source region of the eruption.

\subsection{Event on May 13, 2005}

An erupting filament was observed on 13-May-2005 associated with an M-class flare (start:16:23 UT, peak:16:53 UT).

Evidence of both ejected and surviving material: the filament eruption was associated with a CME comprising a bright core as detected with $\mathrm{LASCO} / \mathrm{C} 2$. The top panel in Fig. 11 displays the $\mathrm{H} \alpha$ images taken from BBSO, showing the filament before eruption (left panel), and the associated flare (middle panel). The right image recorded about $4 \mathrm{~h}$ after the eruption clearly shows the surviving filament (marked with an arrow).

Evidence of internal reconnection (non-PEFR unique): the right image also shows that a two-ribbon flare brackets the surviving filament.

Evidence of observables unique to PEFR model: the middle panel images in Fig. 11 display EIT $195 \AA$ base difference images overplotted with magnetic field contours on top. As is evident from the figure, the dimming regions are outside the source region of eruption and the area of dimming region increases with time and expands outwards. Although no SXR observations were available, the bottom panel in Fig. 11 displays TRACE observations of the eruption. A highly sheared pre-eruption sigmoidal region was visible before the flare (left image, bottom panel). After the eruption (right image) there remain loops that are more sheared towards the north (where the surviving part of the filament resides, see right image in the top panel) than in the southern part of the flaring region.

\section{Discussion and conclusions}

In this paper we have considered multiple cases of partially erupting prominences and have studied their relationship with other CME-associated phenomena such as CME three-part structure, two-ribbon flares, mass flows during eruption, soft $\mathrm{X}$-ray sigmoids and cusps, and coronal dimmings. In order to test the plausibility of the partially-expelled flux rope model of Gibson \& Fan (2006a), we have directly compared these observations to predicted PEFR-model observables. Table 1 shows that all of the events meet our criteria for partial eruption, that is, evidence is observed both for ejected and surviving material, and indirectly for internal reconnection. Moreover, every event show one or both of the PEFR-specific predicted dimmings external to source, and sigmoid to cusp-over-sigmoid transition.

One of the main goals in CME science is to achieve a clear understanding of the pre-CME magnetic field configurations and their evolution. Most CME-initiation models and space weather predictions depend on this. It is therefore worth considering how well our observations distinguish between model predictions. Three competing possibilities, as discussed in Sect. 2, are 1) Model IS (in situ forming flux ropes), 2) Model TE (total eruption of pre-existing flux rope) and 3) Model PEFR (partial eruption of pre-existing flux rope). Table 2 shows the predicted observables of each, and demonstrates the uniqueness of the PEFR model (however, see Mandrini et al. (2007) for an alternate interpretation of dimmings external to source). This, in combination with the results of our observational study, is strong evidence of the plausibility of the PEFR model. This in turn argues that while a magnetic flux rope may well be present prior to eruption, magnetic reconnection appears to be highly significant during eruption. Such reconnection goes beyond merely closing down the magnetic field behind the erupting and expanding flux rope, by playing a crucial role in the bifurcation of the flux rope.

An interesting avenue of future work would be to consider limb observations of partially-erupting prominences. As discussed above, line-of-sight issues make establishing partial eruption of prominences at the limb more difficult. Two-ribbon flares and sigmoids are not visible, and clearly identifying diverging flows is likewise complicated because draining of plasma along the leg of the prominence is an extremely common phenomena. This draining of plasma does not necessarily mean that the prominence has broken while eruption, but could merely arise from plasma sliding back down along field lines that have been pulled radially. The first and rather plausible example of breaking of a prominence at the limb during its eruption was shown by Tripathi et al. (2006b, 2007) based on multi-wavelength observations including the EIT, the LASCO, and MLSO CHIP data. 


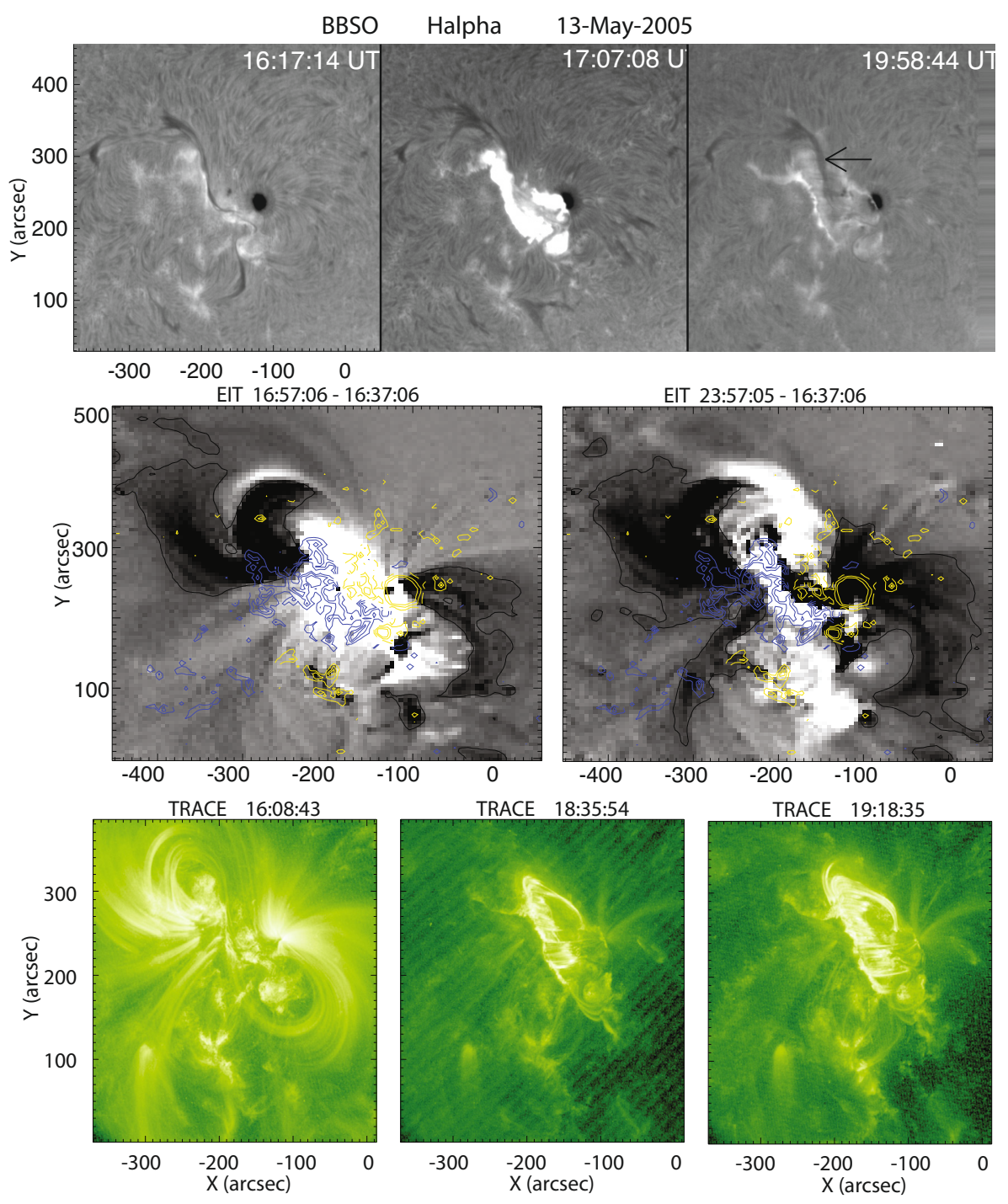

Fig. 11. Top panels: BBSO $\mathrm{H} \alpha$ images showing the filament before eruption (left panel), associated two ribbon flare during eruption (middle panel) and surviving filament in between two ribbons (right panel). The arrow in the last frame locates the surviving filament. Middle panels: base difference images taken by EIT at $195 \AA$ on 13-May-2005, overplotted with MDI magnetic contours, displayed as in Fig. 5. For this event the base image was taken at 16:37 UT (before the flare). Bottom panels: images taken by TRACE on 13-May-2005 at $171 \AA$ before (left panel) and after (middle and right panel) the eruption.

Table 1. Comparison between the PEFR model observables described in Sect. 2 and observations.

\begin{tabular}{|c|c|c|c|c|}
\hline & $\begin{array}{c}\text { Evidence of } \\
\text { ejected } \\
\text { filament material }\end{array}$ & $\begin{array}{l}\text { Evidence of } \\
\text { surviving } \\
\text { filament material }\end{array}$ & $\begin{array}{c}\text { Evidence of } \\
\text { internal reconnections } \\
\text { (not unique to } \\
\text { PEFR model) }\end{array}$ & $\begin{array}{c}\text { Evidence of } \\
\text { internal reconnections } \\
\text { (unique to } \\
\text { PEFR model) }\end{array}$ \\
\hline 29-Apr.-1998 & $\begin{array}{l}\text { white-light CME core; } \\
\text { ejected material visible } \\
\text { in EIT running difference }\end{array}$ & $\begin{array}{l}\text { reformed filament } \\
\text { visible within } \\
\text { two hours }\end{array}$ & $\begin{array}{c}\text { flare ribbons } \\
\text { straddling surviving } \\
\text { filament }\end{array}$ & $\begin{array}{c}\text { sigmoid to } \\
\text { cusp-over-sigmoid (SXR); } \\
\text { dimmings outside source (EUV) }\end{array}$ \\
\hline 17-Aug.-1999 & white-light CME core & $\begin{array}{l}\text { reformed filament } \\
\text { visible within } \\
\text { two and a half hours }\end{array}$ & $\begin{array}{c}\text { flare ribbons } \\
\text { straddling surviving } \\
\text { filament }\end{array}$ & $\begin{array}{c}\text { sigmoid to } \\
\text { cusp-over-sigmoid (SXR); } \\
\text { dimmings outside source (EUV) }\end{array}$ \\
\hline 12-Sep.-2000 & white-light CME core & $\begin{array}{l}\text { reformed filament } \\
\text { visible within } \\
\text { twenty minutes }\end{array}$ & $\begin{array}{c}\text { flare ribbons } \\
\text { straddling surviving } \\
\text { filament } \\
\end{array}$ & $\begin{array}{c}\text { No SXR } \\
\text { observations available; } \\
\text { dimmings outside source (EUV) }\end{array}$ \\
\hline 11-Jun.-2003 & $\begin{array}{l}\text { white-light CME core } \\
\text { four hours }\end{array}$ & $\begin{array}{c}\text { reformed filament } \\
\text { visible within } \\
\text { in erupting filament (He II) }\end{array}$ & $\begin{array}{c}\text { Simultaneous } \\
\text { red and blue shift } \\
\text { dimmings outside source }\end{array}$ & $\begin{array}{c}\text { No SXR } \\
\text { observations available; }\end{array}$ \\
\hline 25-Aug.-2003 & white-light CME core; & $\begin{array}{l}\text { surviving filament } \\
\text { material in TRACE; } \\
\text { reformed next day in } \mathrm{H}-\alpha\end{array}$ & $\begin{array}{l}\text { brightening and diverging } \\
\text { flows at apparent break } \\
\text { point (EUV and white-light) }\end{array}$ & $\begin{array}{c}\text { No SXR } \\
\text { observations available; } \\
\text { dimmings outside source }\end{array}$ \\
\hline 13-May-2005 & white-light CME core & $\begin{array}{l}\text { reformed filament } \\
\text { visible within } \\
\text { four hours }\end{array}$ & $\begin{array}{c}\text { flare ribbons } \\
\text { straddling surviving } \\
\text { filament }\end{array}$ & $\begin{array}{l}\text { No SXR: but EUV } \\
\text { pre-eruption sigmoid } \\
\text { and sheared loops } \\
\text { above surviving filament; } \\
\text { dimmings outside source }\end{array}$ \\
\hline
\end{tabular}


Table 2. Comparing the observables predicted by Model PEFR (see Sect. 2) with Model IS and Model TE.

\begin{tabular}{lccc}
\hline \hline $\begin{array}{l}\text { Predicted observables } \\
\text { (Sect. 2) }\end{array}$ & $\begin{array}{c}\text { Model IS } \\
\text { (In situ forming } \\
\text { flux rope) }\end{array}$ & $\begin{array}{c}\text { Model TE } \\
\text { (Total eruption } \\
\text { of flux rope) }\end{array}$ & $\begin{array}{c}\text { Model PEFR } \\
\text { (Partial eruption } \\
\text { of flux rope) }\end{array}$ \\
\hline 1 (Pre-existing cavity) & & $\sqrt{ }$ & $\sqrt{ }$ \\
2 (Quiescent sigmoid) & $\sqrt{ }$ & & $\sqrt{ }$ \\
3 (Partly erupting filament) & & & $\sqrt{ }$ \\
4 (Partly erupting cavity) & $\sqrt{ }$ & & $\sqrt{ }$ \\
(Flare ribbon surrounding filament) & (Sheared post-eruption loops) & $\sqrt{ }$ & $\sqrt{ }$ \\
7 (Cusp over reformed sigmoid) & & & $\sqrt{ }$ \\
8 (Dimming external to source) & Note $^{a}$ & & \\
\hline
\end{tabular}

${ }^{a}$ See Mandrini et al. (2007) for a possible exception.

It would be worth looking for more such cases, particularly as STEREO observations are now allowing us to consider cases where we would have Earth's-view on-disk observations simultaneous with STEREO EUV limb observations (the SXR observations from the Hinode satellite would enable sigmoid observations as well). Such studies would also resolve line-of-sight ambiguities and so allow a meaningful consideration of significance of reforming cavities.

Another motivation for considering limb observations in future is that writhing motions are best observed at the limb. Such motions are a PEFR-model observable that we have not yet mentioned, due to the kink instability that triggers the eruption in that model (see Fan 2005, for discussion). We did not observe any apparent rotation of filaments during the eruption of any of the disk events studied in this paper. This could be due to the fact that the time scale of the rotation of these filaments is smaller than the cadence of our data. In an independent study using TRACE data Green et al. (2007) studied 7 active region filaments which rotated during eruption. In their study, four events were failed eruptions. It is possible that events in which ejection of material occurs expand more quickly, so that rotation is not visible on the disk. Rotation of material is more easily observed in projection at the limb (see e.g. Liu et al. 2007; and Gibson \& Fan 2008).

Finally, the model for partial eruption of the flux rope can have a substantial significance to space weather predictions. Partial eruption from a region means that magnetic energy is still stored in the surviving twisted field, increasing the likelihood of the region producing homologous flares and CMEs (see e.g., Cheng \& Pallavicini 1987; Chertok et al. 2004). Furthermore, the possibility of partial eruption should be taken into account when studying the geo-effectiveness of CMEs due to a possibility of significant differences between the magnetic field orientations and even topologies of erupted flux rope and that predicted from the CME's source region field configuration (Gibson \& Fan 2008).

Acknowledgements. D.T. and H.E.M. acknowledge the support of STFC. D.T. also acknowledges the support from the High Altitude Observatory (HAO), and thanks HAO for its hospitality during his visit. The National Center for Atmospheric Research is sponsored by the National Science Foundation. This work has benefitted from discussions within the Coronal Prominence Cavity International Team (Leader, Sarah Gibson) of the International Space Science Institute (ISSI), Bern, Switzerland. We thank B. C. Low, Guiliana de Toma, Yuhong Fan, and Scott McIntosh for various useful discussions and comments, and Giuliana de Toma for internal HAO review of this paper. We thank the HAO, EIT, LASCO, TRACE, SXT, GOES teams for providing the data. SoHO is a mission of international collaboration between ESA and NASA. TRACE is a mission of the Stanford-Lockheed Institute for Space Research, and part of the NASA Small Explorer program. We acknowledge the SURF for providing data for use in this publication. We acknowledge observations by Global H $\alpha$ network $(\mathrm{GHN})$ at BBSO and Kanzelhoehe Solar Observatory.

\section{References}

Amari, T., Luciani, J. F., Mikic, Z., \& Linker, J. 1999, ApJ, 518, L57 Amari, T., Luciani, J. F., Aly, J. J., Mikic, Z., \& Linker, J. 2003, ApJ, 585, 1073 Aulanier, G., \& Demoulin, P. 1998, A\&A, 329, 1125

Birn, J., Forbes, T. G., \& Hesse, M. 2006, ApJ, 645, 732

Bothmer, V., \& Rust, D. 1997, Geophys. Monogr., 99, 137

Bothmer, V., \& Schwenn, R. 1997, Annales Geophysicae, 16, 1

Burlaga, L. F. 1988, J. Geophys. Res., 93, 7217

Burlaga, L., Sittler, E., Mariani, F., \& Schwenn, R. 1981, J. Geophys. Res., 86, 6673

Burlaga, L. F., Klein, L., Sheeley, Jr., N. R., et al. 1982, Geophys. Res. Lett., 9, 1317

Canfield, R. C., Hudson, H. S., \& Pevtsov, A. A. 2000, IEEE Trans. Plasma Sci., 28,1786

Chen, J., Howard, R. A., Brueckner, G. E., et al. 1997, ApJ, 490, L191

Cheng, C.-C., \& Pallavicini, R. 1987, ApJ, 318, 459

Chertok, I. M., Grechnev, V. V., Hudson, H. S., \& Nitta, N. V. 2004, J. Geophys. Res. (Space Physics), 109, 2112

Chifor, C., Mason, H. E., Tripathi, D., Isobe, H., \& Asai, A. 2006, A\&A, 458, 965

Chifor, C., Tripathi, D., Mason, H. E., \& Dennis, B. R. 2007, A\&A, 472, 967

Dere, K. P., Brueckner, G. E., Howard, R. A., Michels, D. J., \& Delaboudinière, J. P. 1999, ApJ, 516, 465

Fan, Y. 2001, ApJ, 554, L111

Fan, Y. 2005, ApJ, 630, 543

Fisher, R. R., \& Poland, A. I. 1981, ApJ, 246, 1004

Gibson, S. E., \& Fan, Y. 2006a, ApJ, 637, L65

Gibson, S. E., \& Fan, Y. 2006b, J. Geophys. Res. (Space Physics), 111, 12103

Gibson, S. E., \& Fan, Y. 2008, J. Geophys. Res. (Space Physics), 113, 9103

Gibson, S. E., Fletcher, L., Del Zanna, G., et al. 2002, ApJ, 574, 1021

Gibson, S. E., Fan, Y., Mandrini, C., Fisher, G., \& Demoulin, P. 2004, ApJ, 617, 600

Gibson, S. E., Fan, Y., Török, T., \& Kliem, B. 2006a, Space Sci. Rev., 124, 131

Gibson, S. E., Foster, D., Burkepile, J., de Toma, G., \& Stanger, A. 2006b, ApJ, 641,590

Gilbert, H. R., Holzer, T. E., Burkepile, J. T., \& Hundhausen, A. J. 2000, ApJ, 537,503

Gloeckler, G., Fisk, L. A., Hefti, S., et al. 1999, Geophys. Res. Lett., 26, 157

Green, L. M., Harra, L. K., Matthews, S. A., \& Culhane, J. L. 2001, Sol. Phys., 200, 189

Green, L. M., Kliem, B., Török, T., van Driel-Gesztelyi, L., \& Attrill, G. D. R. 2007, Sol. Phys., 246, 365

Hundhausen, A. 1999, in The many faces of the sun: a summary of the results from NASA's Solar Maximum Mission., ed. K. T. Strong, J. L. R. Saba, B. M. Haisch, \& J. T. Schmelz, 143

Illing, R. M. E., \& Hundhausen, A. J. 1985, J. Geophys. Res., 90, 275 Janse, A. M., \& Low, B. C. 2007, A\&A, 472, 957

Lin, J., Forbes, T. G., Isenberg, P. A., \& Demoulin, P. 1998, ApJ, 504, 1006 Lites, B. W., Low, B. C., Martinez Pillet, V., et al. 1995, ApJ, 446, 877 Liu, R., Alexander, D., \& Gilbert, H. R. 2007, ApJ, 661, 1260

López Fuentes, M. C., Demoulin, P., Mandrini, C. H., \& van Driel-Gesztelyi, L. 2000, ApJ, 544, 540 
Low, B. C. 1996, Sol. Phys., 167, 217

Low, B. C. 1999, in Magnetic Helicity in Laboratory and Space Plasmas (AGU Publications), ed. Canfield et al., 25

Manchester, IV, W., Gombosi, T., DeZeeuw, D., \& Fan, Y. 2004, ApJ, 610, 588 Mandrini, C. H., Démoulin, P., Schmieder, B., Deng, Y. Y., \& Rudawy, P. 2002, A\&A, 391, 317

Mandrini, C. H., Nakwacki, M. S., Attrill, G., et al. 2007, Sol. Phys., 244, 25 Maričić, D., Vršnak, B., Stanger, A. L., \& Veronig, A. 2004, Sol. Phys., 225, 337 Martin, S. F., \& McAllister, A. H. 1995, International Astronomical Union, 497 Moore, R. L., Schmieder, B., Hathaway, D. H., \& Tarbell, T. D. 1997, Sol. Phys., 176,153

Plunkett, S. P., Vourlidas, A., Šimberová, S., et al. 2000, Sol. Phys., 194, 371

Priest, E. R. 1984, Solar magneto-hydrodynamics, Geophysics and Astrophysics Monographs (Dordrecht: Reidel)

Priest, E. R., Hood, A. W., \& Anzer, U. 1989, ApJ, 344, 1010

Qiu, J., Howard, T. A., \& Yurchyshyn, V. B. 2007, ApJ, in press

Reinard, A. 2005, ApJ, 620, 501

Rust, D. M. 2003, Adv. Space Res., 32, 1895

Rust, D. M., \& Kumar, A. 1994, Sol. Phys., 155, 69
Rust, D. M., Anderson, B. J., Andrews, M. D., et al. 2005, ApJ, 621, 524

Skoug, R. M., Bame, S. J., Feldman, W. C., et al. 1999, Geophys. Res. Lett., 26, 161

Srivastava, N., Schwenn, R., \& Stenborg, G. 1999, in 8th SOHO Workshop: Plasma Dynamics and Diagnostics in the Solar Transition Region and Corona, ed. J.-C. Vial, \& B. Kaldeich-Schü, ESA SP-446, 621

Su, Y. N., Golub, L., van Ballegooijen, A. A., \& Gros, M. 2006, Sol. Phys., 236, 325

Taylor, J. B. 1974, Phys. Rev. Lett., 33, 1139

Tokman, M., \& Bellan, P. M. 2002, ApJ, 567, 1202

Tripathi, D. 2006, J. Astrophys. Astron., 27, 193

Tripathi, D., Bothmer, V., \& Cremades, H. 2004, A\&A, 422, 337

Tripathi, D., Isobe, H., \& Mason, H. E. 2006a, A\&A, 453, 1111

Tripathi, D., Solanki, S. K., Schwenn, R., et al. 2006b, A\&A, 449, 369

Tripathi, D., Solanki, S. K., Mason, H. E., \& Webb, D. F. 2007, A\&A, 472, 633

van Ballegooijen, A. A. 2004, ApJ, 612, 519

Zhang, J., \& Dere, K. P. 2006, ApJ, 649, 1100

Zhang, J., Dere, K. P., Howard, R. A., Kundu, M. R., \& White, S. M. 2001, ApJ, 559,452 